Supporting information

\title{
Non-flammable and High-Voltage-Tolerated Polymer Electrolyte achieving High Stability and Safety in 4.9 V-class Lithium Metal Battery
}

Jie Liu, Xiaowei Shen, Jinqiu Zhou, Mengfan Wang, Chaoqun Niu, Tao Qian* and Chenglin Yan*

Soochow Institute for Energy and Materials InnovationS, College of Energy, Key Laboratory of Advanced Carbon Materials and Wearable Energy Technologies of Jiangsu Province, Soochow University, Suzhou 215006, China.

\section{Corresponding Author}

E-mail: tqian@suda.edu.cn (T. Q.); c.yan@suda.edu.cn (C. Y.) 


\section{EXPERIMENTAL PROCEDURES}

Preparation of HVTPE in batteries: $20 \mathrm{wt} \%$ LiDFOB was dissolved in FEC solution, and the ultrasonic process was conducted to form a homogeneous mixture. Subsequently, $1 \mathrm{wt} \% \mathrm{Sn}(\mathrm{Oct})_{2}$ as initiator was added in, following by well stirring, and the HVTPE precursor solution was obtained. The whole preparation process was carried out in Ar atmosphere. The LNMO cathode was prepared by mixing LNMO powder, carbon black and PVDF at a weight ratio of 8:1:1 in NMP to form homogeneous slurry, then following by casting on Al foil and vacuum drying. The average areal mass loading of LNMO was $2.9 \mathrm{mg} \mathrm{cm}^{-2}$. Subsequently, the prepared precursor solution was incorporated into a cellulose separator and 2032-type cells were assembled. Later, the batteries were kept constantly at $80{ }^{\circ} \mathrm{C}$ for $24 \mathrm{~h}$ and $100{ }^{\circ} \mathrm{C}$ for another $24 \mathrm{~h}$ to complete Li/HVTPE/LNMO cell. The Li/HVTPE/LCO cell was assembled in a similar method with Li/HVTPE/LNMO cell and the average areal mass loading of LCO was $3.2 \mathrm{mg} \mathrm{cm}^{-2}$.

Preparation of PEO-GPE in batteries: The PEO-GPE slurry was prepared by dissolving PEO powder and $\mathrm{LiDFOB}(\mathrm{O} / \mathrm{Li}$ ratio of $8 / 1)$ in acetonitrile and vigorous mixing to yield a uniform dispersion. Then, the slurry was incorporating into a cellulose separator following by evaporating excess solvent. Subsequently, Li/PEO-SPE/LNMO cell was assembled in a 2032-type cell as contrast, where $5 \mu \mathrm{L} \mathrm{EC/DEC} \mathrm{(1:1} \mathrm{in} \mathrm{vol)} \mathrm{was} \mathrm{added} \mathrm{to} \mathrm{improve} \mathrm{the} \mathrm{interface.}$

Characterizations: Proton and carbon nuclear magnetic resonance $\left({ }^{1} \mathrm{H}\right.$ and $\left.{ }^{13} \mathrm{C} \mathrm{NMR}\right)$ spectra were taken on Agilent (DD2-600, America) with proton frequency of $600 \mathrm{MHz}$. Deuterated dimethyl sulfoxide (DMSO- $d_{6}$ ) was used as the solvent. The morphologies of cycled Li foils and LCO electrodes were observed by field emission scanning electron microscopy (SEM, SU8010, Japan) operated at $5.0 \mathrm{kV}$. The Young's modulus of HVTPE was studied using an AFM instrument (Bruker DIMENSION ICON with a Nanoscope V controller) by measuring normal deflection signal change of the cantilever during the tip loading and unloading at the sample surface. The loading curve describes the force as a function of sample deformation when the tip moves towards HVTPE, and conversely, the unloading curve suggests the tip moves away from the sample. The parameters of AFM tips were $40 \mathrm{~N} / \mathrm{m}$. The X-ray photoelectron spectroscopy (XPS) measurements were carried out in an ultra-high vacuum ESCALAB 250 set-up equipped with a monochromatic Al Ka X-ray source (1486.6 eV; anode operating at $15 \mathrm{kV}$ and $20 \mathrm{~mA})$.

In-situ Optical Microscopic Observations: In-situ observations were carried out in the 
home-made optical cell. PEO-GPE covered Li and HVTPE covered Li were respectively used as working electrodes. After injecting electrolyte, the cell was well-sealed. The assembly of the optical cell was performed in an Argon-filled glove box with water and oxygen content less than $0.1 \mathrm{ppm}$. Optical microscope with a fitted charge coupled device (CCD) camera was applied to shoot the Li deposition process.

Electrochemical measurements: Symmetric cells with two identical Li foil were assembled using HVTPE or PEO-GPE to study the Li stripping/plating processes. The charge/discharge process was monitored in a galvanostatic mode using a CT2001A cell test instrument (Wuhan LAND electronics Co., Ltd.). Li/HVTPE/SS asymmetrical cell was assembled to evaluate the electrochemical stable window of HVTPE.

The ionic conductivity of HVTPE and PEO-GPE was determined via an AC technique. In order to achieve closed interfacial contact between HVTPE and stainless steel, and minimize the interfacial resistance, in-situ polymerization method was employed in stainless steel symmetric cell. FEC solution contained 1.0 M LiDFOB and $1 \mathrm{wt} \% \mathrm{Sn}(\mathrm{Oct})_{2}$ was incorporated into a cellulose separator, which was sandwiched by two sheets of stainless steel. Later, the batteries were kept constantly at $80^{\circ} \mathrm{C}$ for $24 \mathrm{~h}$ and $100{ }^{\circ} \mathrm{C}$ for another $24 \mathrm{~h}$ to complete the polymerization of FEC. Data were acquired using CHI660E electrochemical workstation (Shanghai Chenhua instrument Co., Ltd) with AC voltage amplitude of $10 \mathrm{mV}$ over the frequency range from $0.1 \mathrm{~Hz}$ to $1 \mathrm{MHz}$. The ionic conductivity $(\sigma)$ of polymer electrolyte was calculated by using the following equation:

$$
\sigma=\frac{I}{S R}
$$

where "I" presents the thickness of polymer electrolyte $(\sim 70 \mu \mathrm{m})$, "S" is the contact area between electrode and electrolyte $\left(2 \mathrm{~cm}^{2}\right)$, and " $\mathrm{R}$ " corresponds to the bulk resistance of the polymer electrolyte.

Theoretical calculation: DFT calculations were performed at the B3LYP/6-31+G(d, p) level using Gaussian 09, including the geometrical structures and the vibrational modes. 


\section{Figures:}

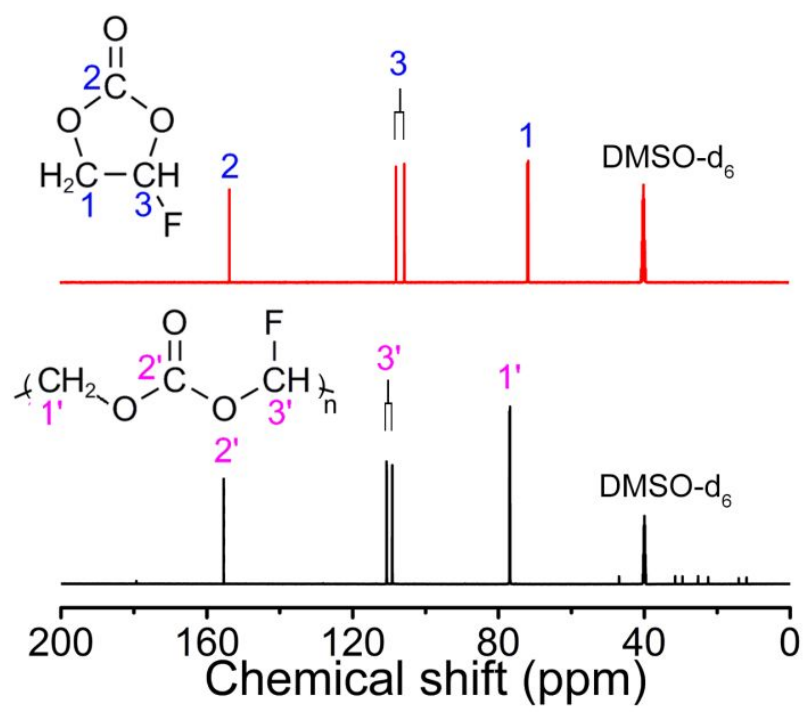

Figure S1. ${ }^{1} \mathrm{C}$ NMR spectra of FEC and PFEC in DMSO- $d_{6}$. The ${ }^{1} \mathrm{C}$ chemical shifts imply the opening of the FEC ring. 


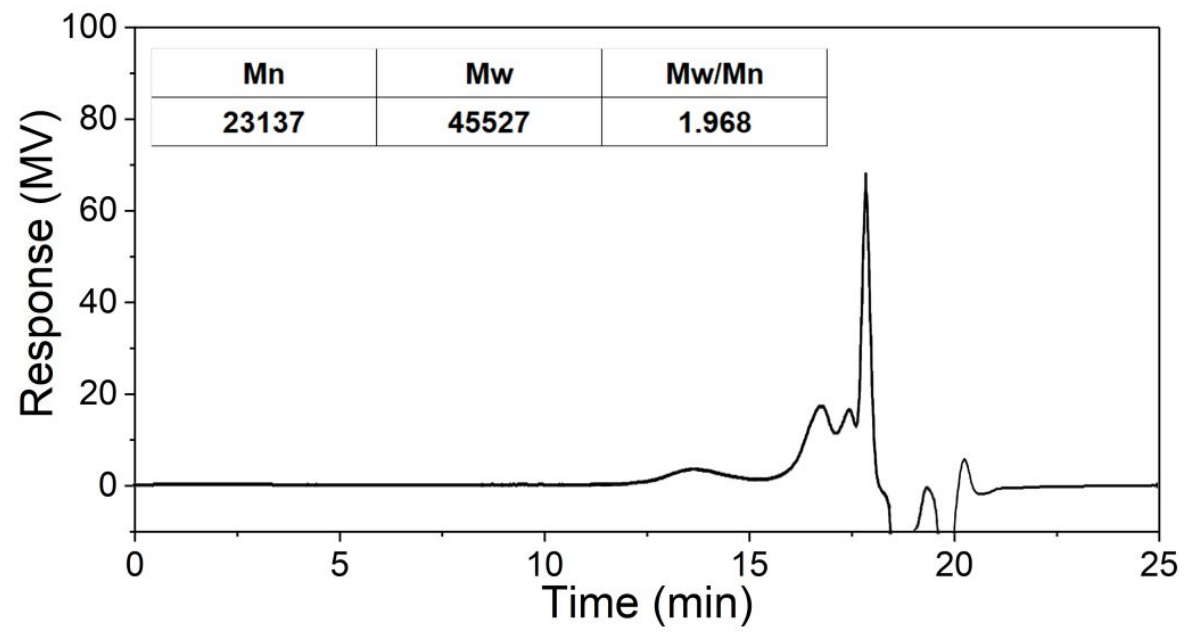

Figure S2. GPC result of PFEC, showing the weight-average molecular weight of $\sim 4.55 \times 10^{4}$. 


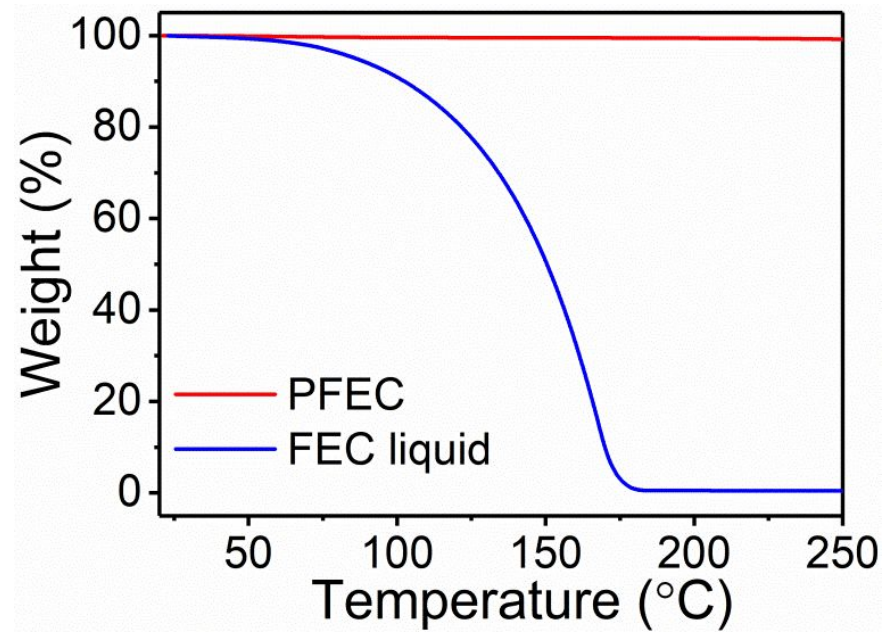

Figure S3. TG curves of FEC liquid and PFEC. 


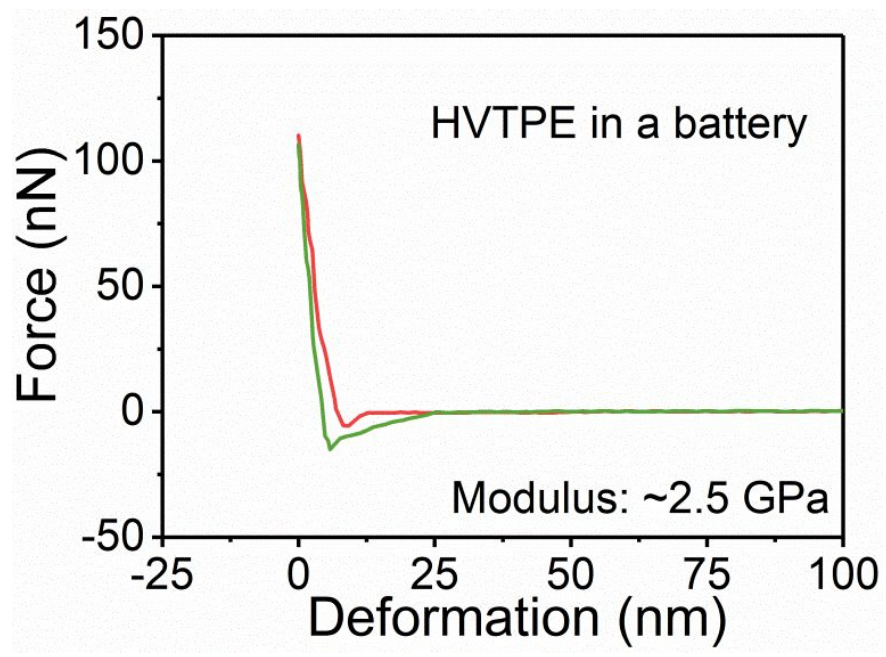

Figure S4. The indentation curves of HVTPE which polymerized in batteries. 


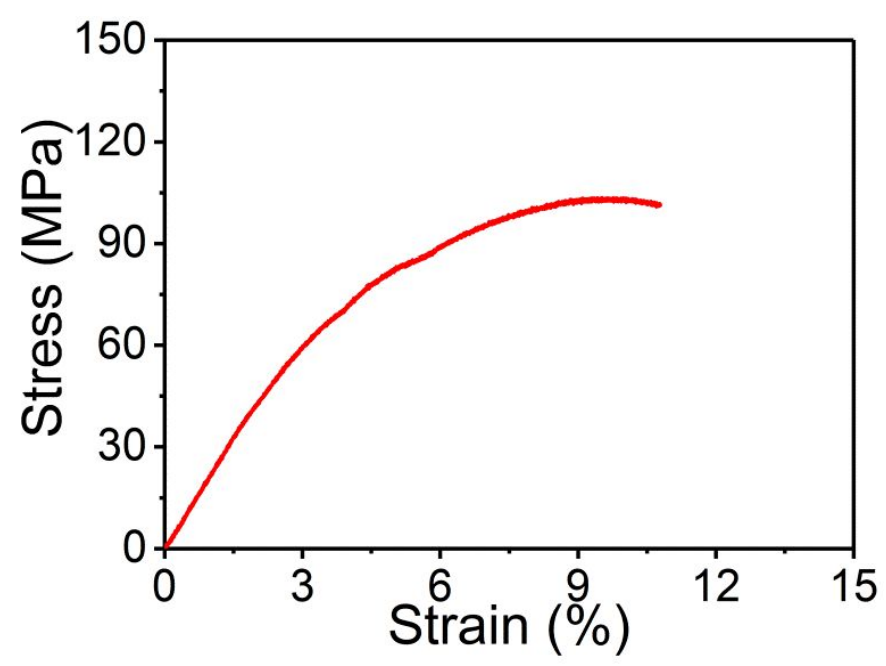

Figure S5. Tensile stress-strain curve of PFEC, showing the ultimate tensile strain of $\sim 9.6 \%$ and tensile strength of $\sim 103 \mathrm{MPa}$. 


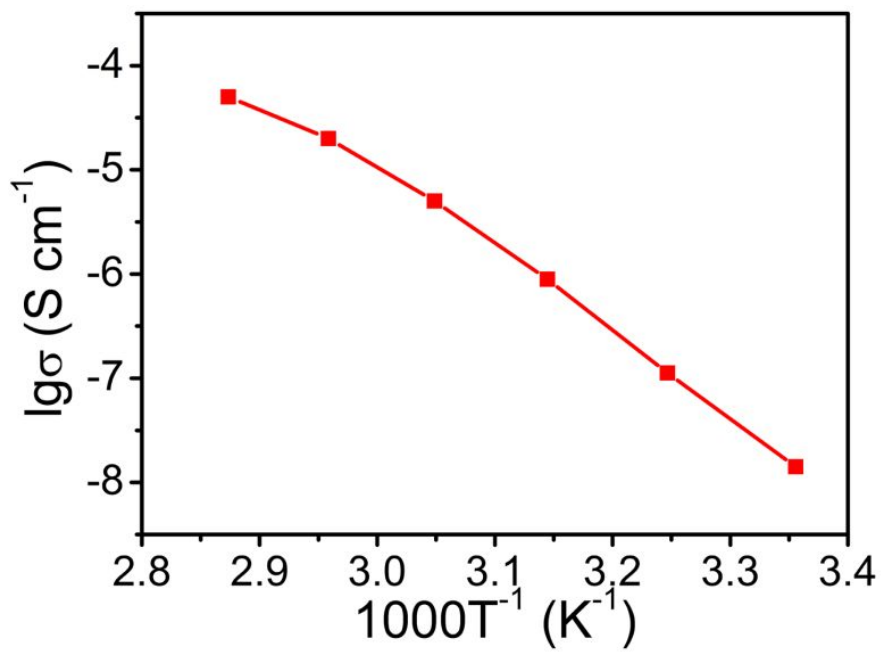

Figure S6. Temperature dependent ionic conductivity of PEO-SPE, showing the very low ionic conductivity at ambient temperature. 


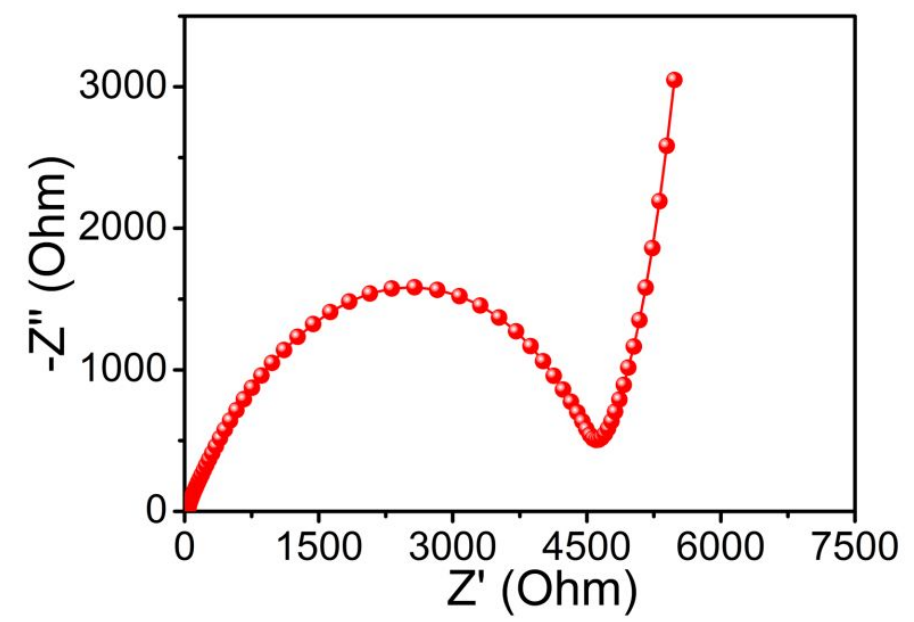

Figure S7. EIS spectra of the Li/PEO-SPE/Li symmetric cells, demonstrating the tremendous interfacial resistance between $\mathrm{Li}$ and electrolyte. 


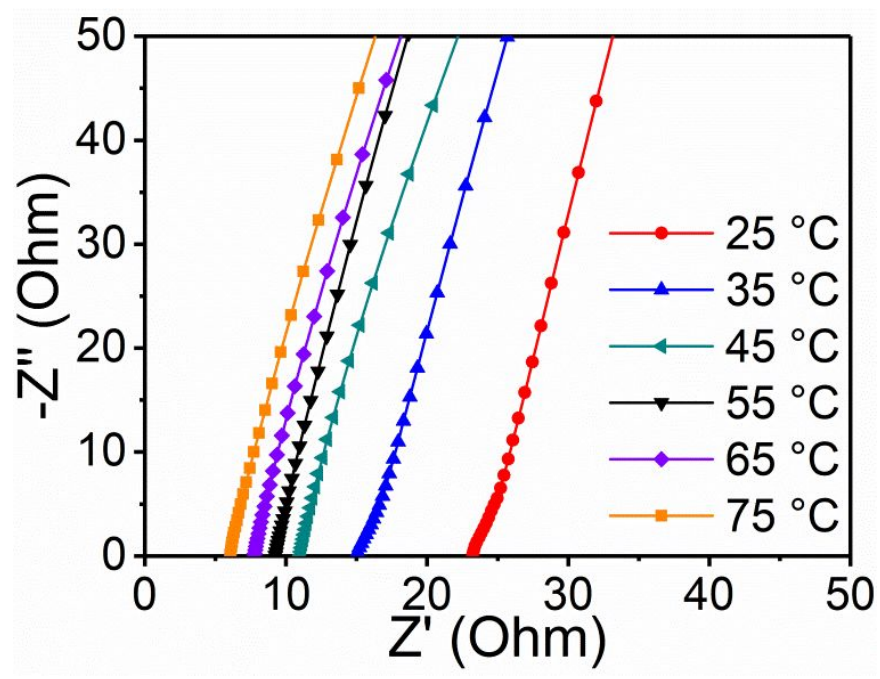

Figure S8. The impedance spectra of SS/HVTPE/SS symmetric cell at the temperature range of $25^{\circ} \mathrm{C} \sim 75^{\circ} \mathrm{C}$. 


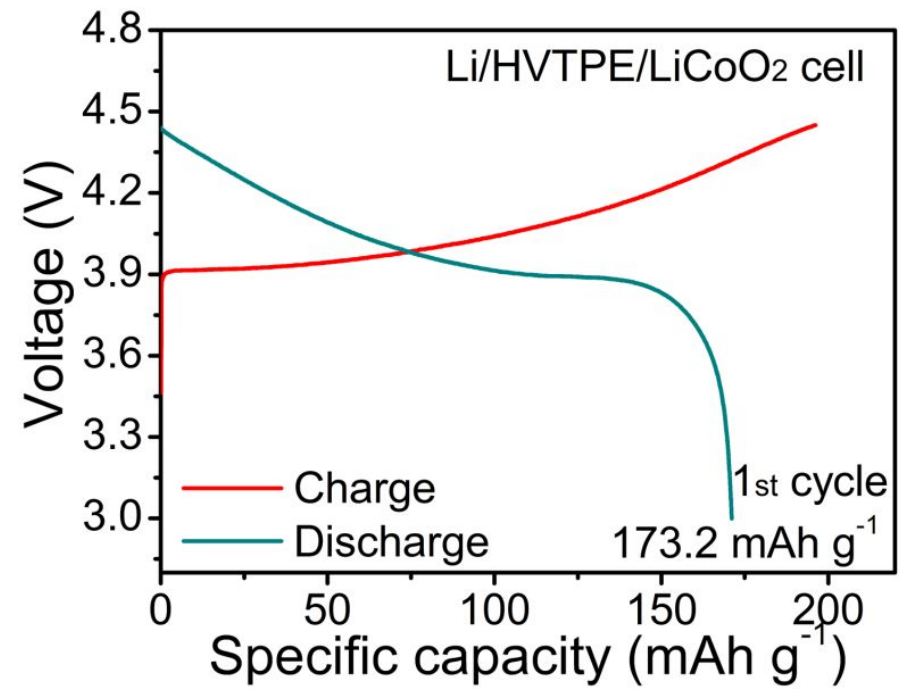

Figure S9. The charging/discharging curves of Li/HVTPE/LCO cell at C/20 rate with the voltage window of $2.5-4.45 \mathrm{~V}$. 


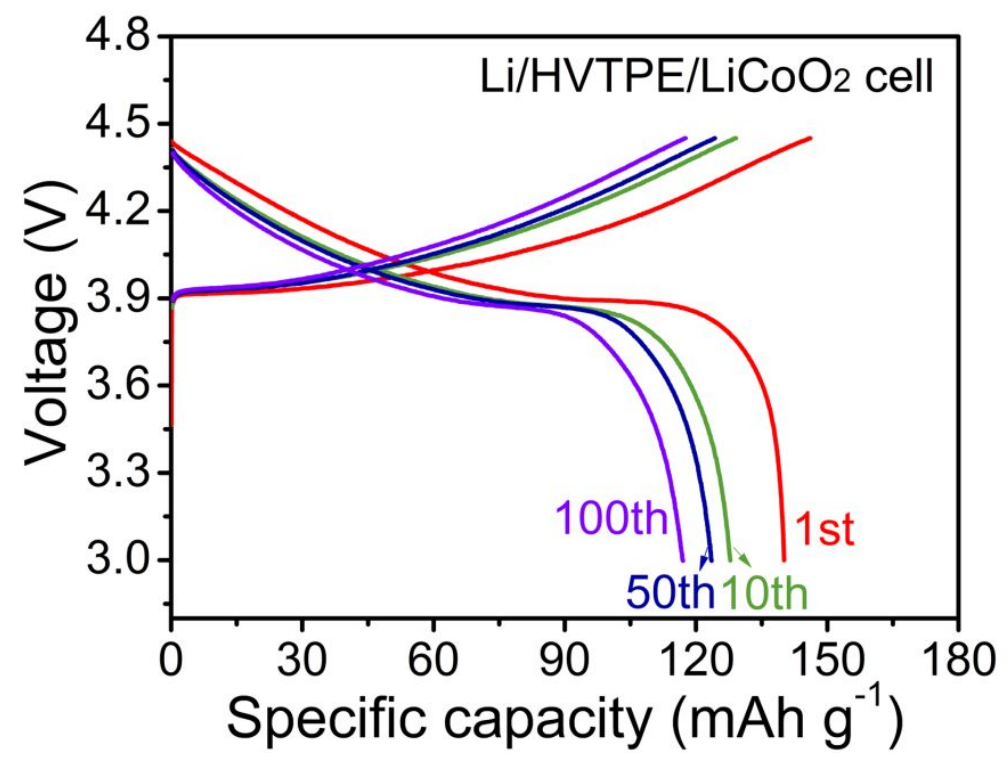

Figure S10. The corresponding charging/discharging voltage curves of Li/HVTPE/LCO cell in 1st, 10 th, 50th and 100th cycles at room temperature. 


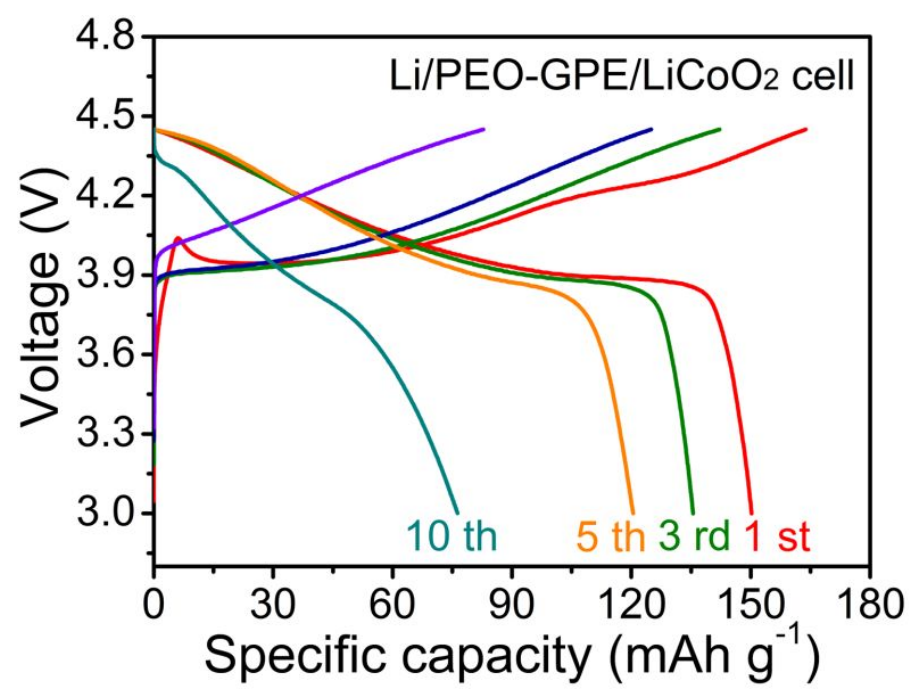

Figure S11. The compared charging/discharging voltage curves of Li/PEO-GPE/LCO cell, demonstrating a rapid capacity decay. 


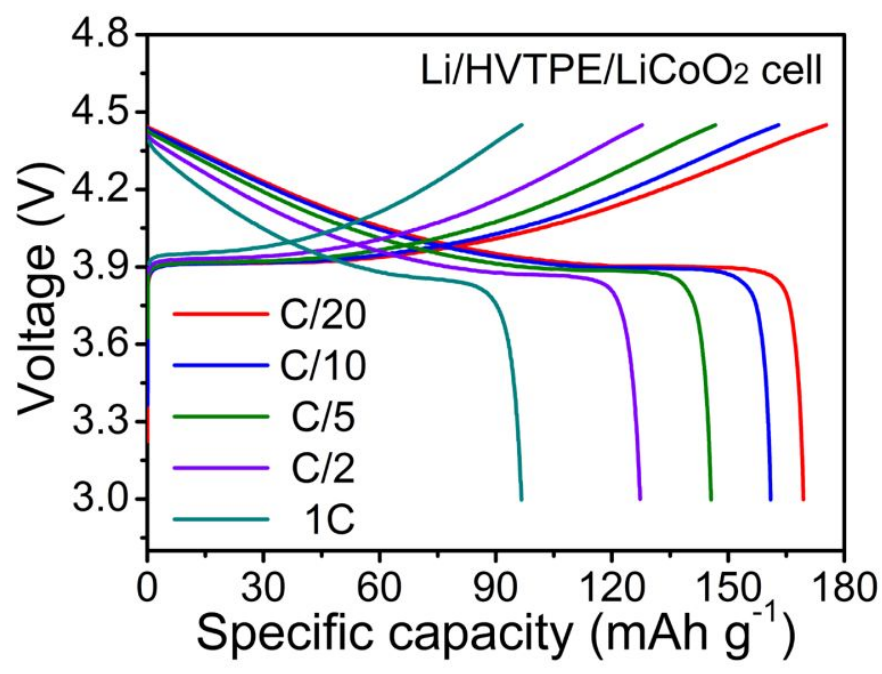

Figure S12. Charging/discharging voltage curves of the $4.45 \mathrm{~V}$-class Li/HVTPE/LCO cell at varied current rates. 


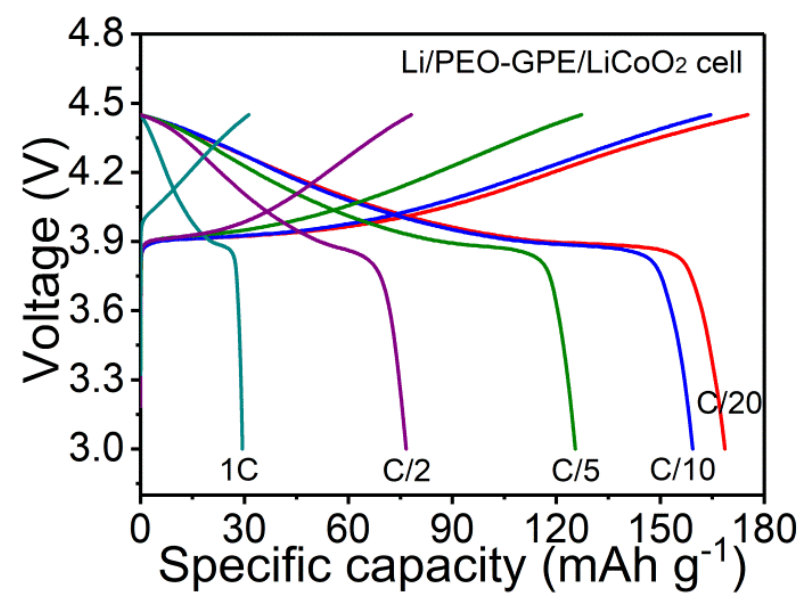

Figure S13. Charging/discharging voltage curves of the Li/PEO-GPE/LCO cell at varied current rates. 

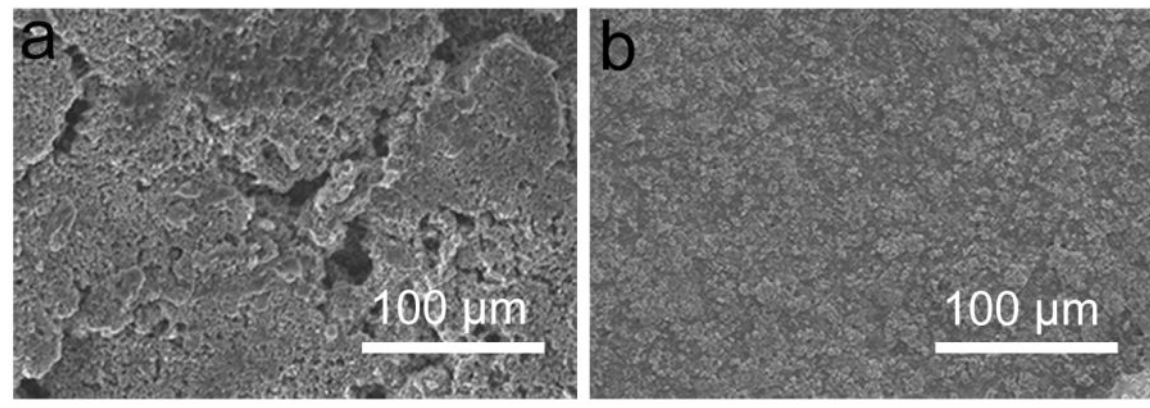

Figure S14. The surface morphologies of cycled LCO cathodes retrieved from (a) Li/PEO-GPE/LCO and (b) Li/HVTPE/LCO cell. 


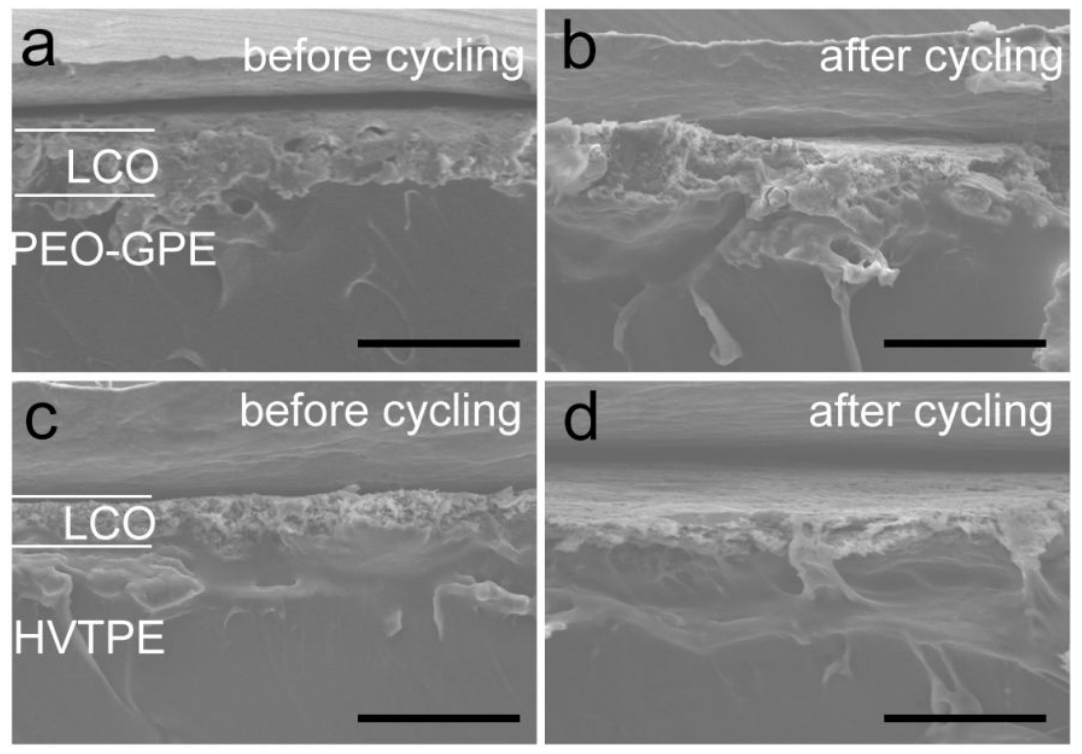

Figure S15. The cathode/electrolyte interface of Li/PEO-GPE/LCO cell (a) before and (b) after cycling. The cathode/electrolyte interface of Li/HVTPE/LCO cell (c) before and (d) after cycling. 


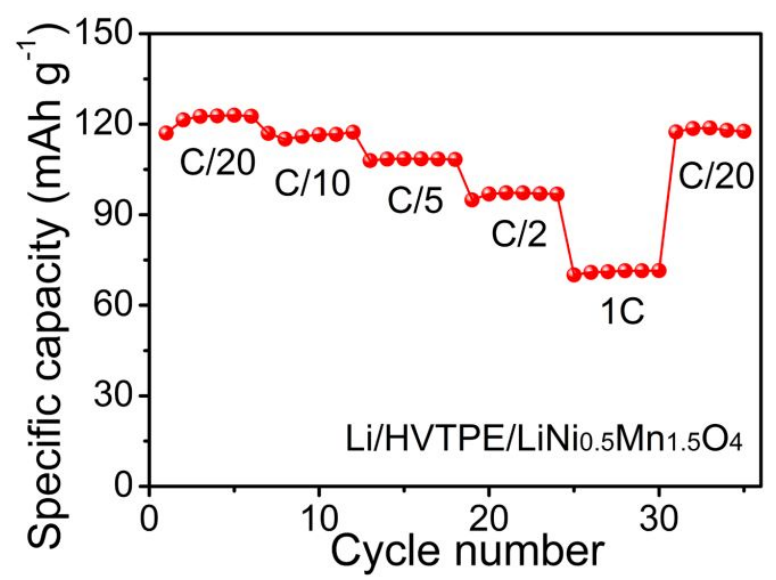

Figure S16. The rate performance of Li/HVTPE/LNMO battery. 


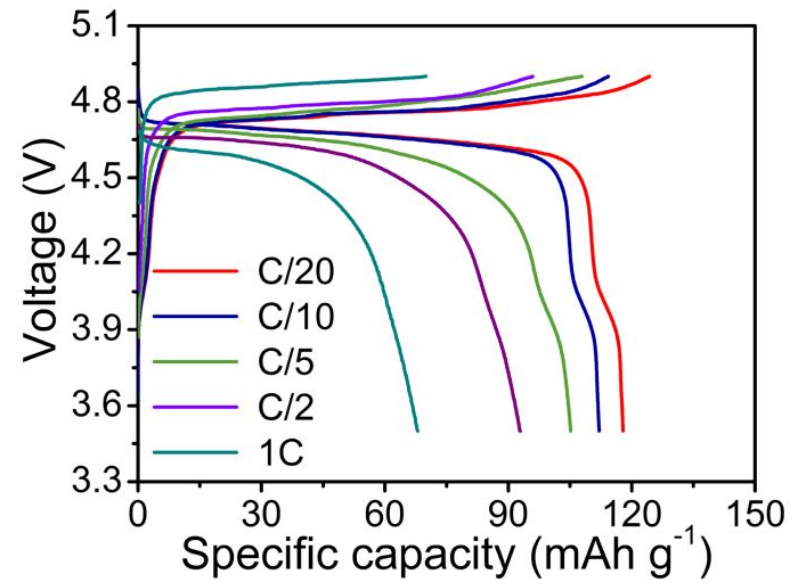

Figure S17. Charging/discharging voltage curves of $4.9 \mathrm{~V}$-class Li/HVTPE/LNMO cell at varied current rates between $\mathrm{C} / 20-1 \mathrm{C}$. 


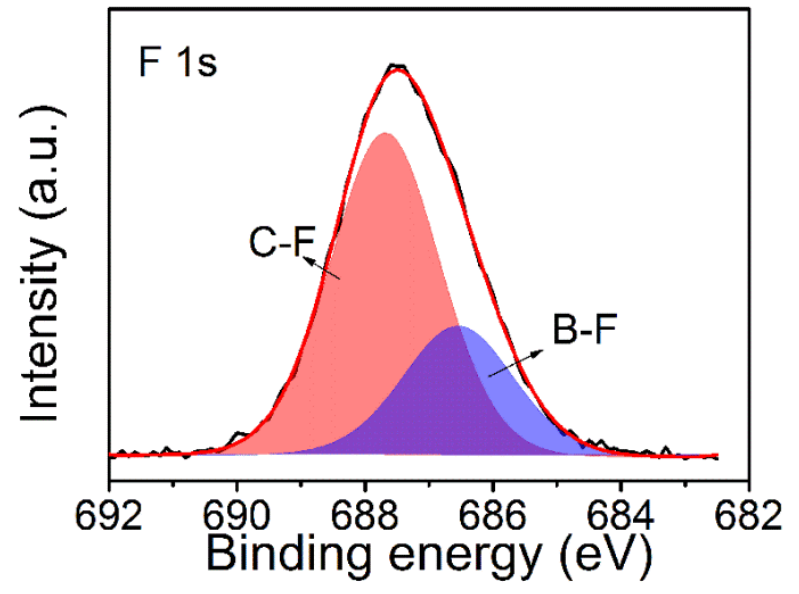

Figure S18. F1s spectra of Li anodes before the cell was cycled. 

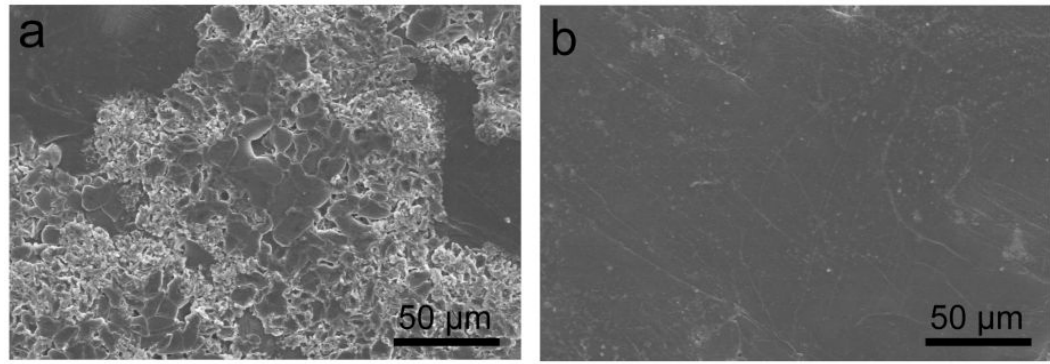

Figure S19. SEM images of cycled Li metals retrieved from (a) Li/PEO-GPE/Li and (b) Li/HVTPE/Li symmetric cells. 
Table S1 HOMO and LUMO energies of different monomers or units.

\begin{tabular}{ccc}
\hline HOMO energies $(\mathbf{e V})$ & LUMO energies (eV) \\
FEC & -8.40 & -0.24 \\
PEO unit & -8.90 & -0.37 \\
Ring-opened EC & -7.58 & 0.01 \\
Ring-opened FEC & -8.16 & 0.13 \\
\hline
\end{tabular}

